\title{
Differences between women and men
}

\section{in use of antithrombotic drugs in patients with stroke and atrial fibrillation}

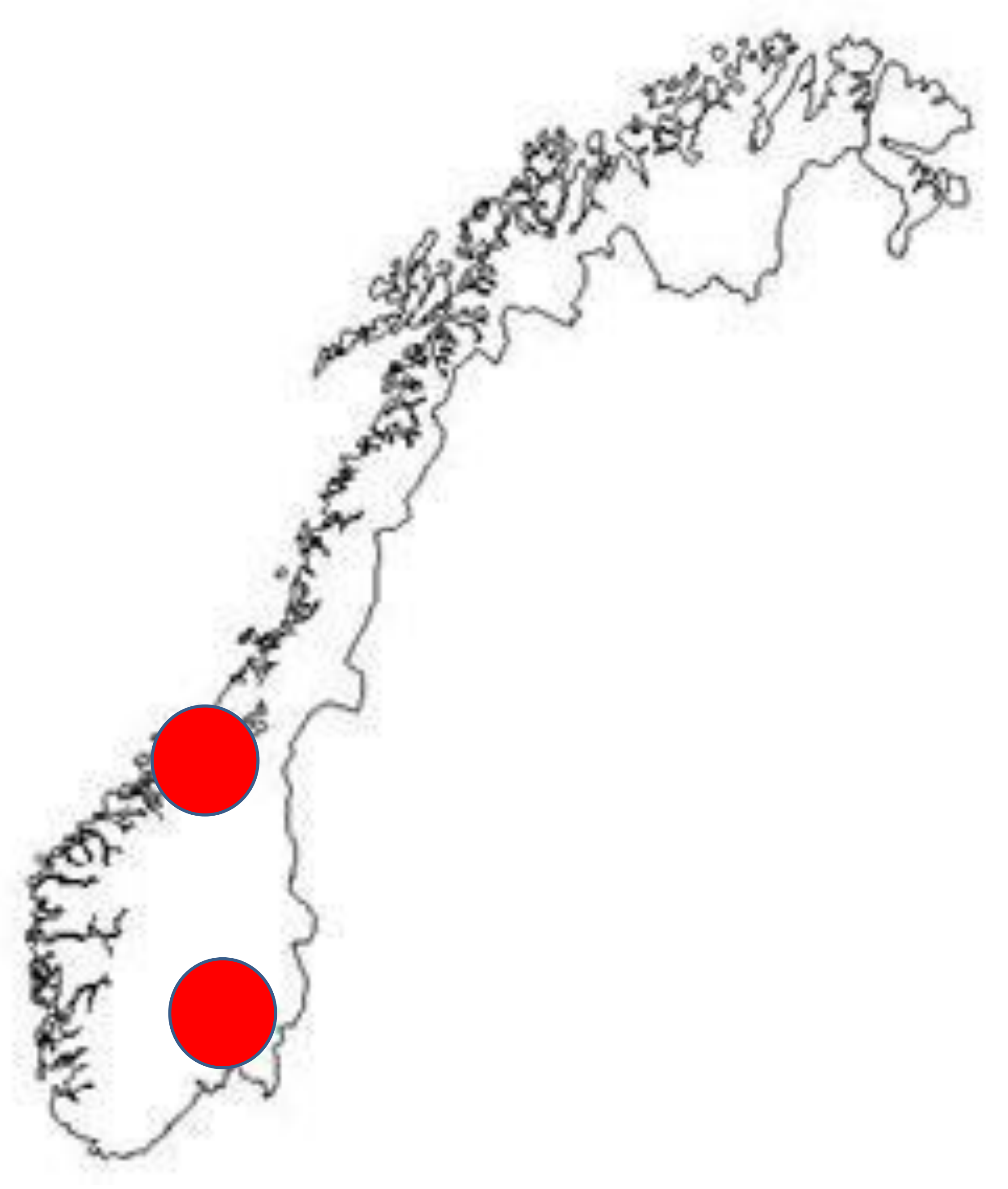

Women with AF were less likely than men to be on treatment with new oral anticoagulants prior to the stroke.

Hege Ihle-Hansen, MD, PhD, Department of Medicine, Oslo University Hospital, Ullevả,, Oslo, Norway
se Charlotte Sandset, MD, PhD, Department of Neurology, OSlo University Hospital, Ullevaál, OSlo, Norway
th-Stephani, Norwegian Stroke Registry, NTNU, Norrvegian University of Science and Technology, Trondheim, Norway
Jft, PhD, Norwegian Stroke Registry, NTNU, Norwegian University of Science and Technology, Trondheim, Norway

\section{Introduction}

Women who suffer stroke are older than men, have more cardioembolic aetiology including atrial fibrillation (AF) and suffer from more severe strokes.

We aimed to investigate whether the use of antithrombotic drugs is different in women and men with stroke and AF.

\section{Methods}

We used data from the Norwegian Stroke Registry 2016 and extracted people with detected AF, both known and detected during the hospital stay.

Using the Chi-square test we compared the use of different antithrombotic drugs prior to stroke and upon discharge.

\section{Results}

Out of 8650 patients, 2290 (26.5\%) had AF. There were significantly more AF among women than men $(27.7$ vs $25.5 \%, p=0.02)$.

On admission, there were no differences between women and men in the use of aspirin (31.2 vs $32.1 \%, p=0.495)$, clopidorgrel ( 1.9 vs $2.5 \%, p=0.327$ ) or warfarin

(21.8 vs $23.6, p=0.30)$. Women were less likely to be on treatment with other anticoagulants ( 19.4 vs $23.4 \%, p=0.017$ ).

At discharge, there were no differences in the use of either warfarin or other anticoagulants, however, men were more often on treatment with aspirin (17.1 vs $21.8 \%,<0.001)$ and clopidogrel (1.8 vs $4.1 \%, p=0.002)$.

\section{Conclusion}

The proportion of $\mathrm{AF}$ is higher among women than among men. Women were less likely than men to be treated with other anticoagulants prior to the stroke, 